\title{
PRESSÃO ARTERIAL SISTÓLICA E EXCREÇÃO URINÁRIA DE PROTEÍNAS DE CÃES IDOSOS SADIOS OU COM DOENÇA RENAL CRÔNICA TRATADOS COM O ANTIOXIDANTE N-ACETILCISTEÍNA
}

\author{
GALVÃO, André Luiz Baptista ${ }^{1}$ \\ CARVALHO, Marileda Bonafim ${ }^{2}$
}

\begin{abstract}
Recebido em: 2015.04 .27
Aprovado em: 2015.11.27

ISSUE DOI: $10.3738 / 21751463.1471$

RESUMO: Os mecanismos fisiopatológicos das doenças renais incluem lesão secundária e perda celular relacionadas ao acúmulo de espécies reativas do oxigênio (ERO). A progressão da doença renal crônica (DRC) também envolve a hipertensão arterial sistêmica (HAS), proteinúria associadas à ativação crônica do sistema reninaangiotensina-aldosterona (SRAA) e ao estresse oxidativo. A intervenção terapêutica com os chamados antioxidantes pode conferir proteção renal por meio da remoção de substâncias reativas. A N-acetilcisteína (NAC) é um antioxidante que tem sido empregado para tal fim em casos de isquemia renal, exposição a fármacos nefrotóxicos e na insuficiência renal crônica. No presente trabalho, o objetivo proposto foi avaliar os efeitos do tratamento com NAC sobre a pressão arterial sistólica (PAS) e na razão proteína/creatinina urinária (U-P/C) de cães idosos sadios e de cães com DRC naturalmente adquirida, clinicamente estáveis. Foram avaliados quatro grupos de cães idosos ( 9 a 15 anos), compreendendo o normal controle $(\mathrm{N}-\mathrm{C} ; \mathrm{n}=4)$, normal tratado $(\mathrm{N}-\mathrm{T} ; \mathrm{n}=5)$, DRC controle $(\mathrm{DRC}-\mathrm{C} ; \mathrm{n}=$ 5) e DRC tratado (DRC-T; $n=4)$. Os cães dos grupos N-T e DRC-T receberam como tratamento único a NAC na dose de $10 \mathrm{mg} / \mathrm{kg}$, V.O., b.i.d, durante 60 dias. Os cães dos grupos N-C e DRC-C não receberam qualquer tipo de tratamento. Os parâmetros PAS e U-P/C foram avaliados nos dias zero (basal), 15, 30, 45 e 60. Os dados foram submetidos a ANOVA e teste Tukey-Kramer $(\alpha=0,05)$. A NAC não exerceu efeito nenhum sobre a U-P/C, entretanto, na PAS de cães idosos sadios houve uma tendência a redução a partir de 15 dias de tratamento. Concluiuse que a NAC promoveu em cães idosos sadios uma tendência de diminuição da PAS.
\end{abstract}

Palavras-chave: Creatinina; Hipertensão; Proteinúria

\section{SYSTOLIC BLOOD PRESSURE AND URINARY PROTEINS EXCRETION OF OLDER DOGS HEALTHY OR WITH CHRONIC DISEASE TREATED WITH ANTIOXIDANT N-ACETYLCYSTEINE}

\begin{abstract}
SUMMARY: The pathophysiological mechanisms of chronic kidney disease (CKD) include secondary injury and cells related to the accumulation of reactive oxygen species (ROS). The progression of CKD is also involves arterial hypertension (HBP) and proteinuria associated with chronic activation of the rennin-angiotensin-aldosterone (RAAS) and oxidative stress. The therapeutic intervention with antioxidants called may confer renal protection through the removal reactive substances. N-acetylcystine (NAC) is an antioxidant that has been used for such a purpose in cases of renal ischemia and exposure to nephrotoxic drugs and chronic renal failure. The objective of this study was to the effects of treatment with NAC concerning systolic arterial pressure (SBP) and ratio urine protein/creatinine (U-P/C) of older dogs healthy and dogs with naturally acquired CKD, clinically stable. Have been assessed four groups of older dogs (9-15 years), comprising normal control $(\mathrm{N}-\mathrm{C}, \mathrm{n}=4)$, normal treated $(\mathrm{N}-\mathrm{T}, \mathrm{n}=5)$, CKD control (CKD-C, $\mathrm{n}=5)$ and $\mathrm{CKD}$ treated $(\mathrm{CKD}-\mathrm{T}, \mathrm{n}=4)$. The dogs from group N-T and CKD-T have received as a single treatment with NAC at a dose of $10 \mathrm{mg} / \mathrm{Kg}$, po, b.i.d., for 60 days. The dogs from group N-C and CKD-C have not received any type of treatment. The parameters SBP and U-P/C have been evaluated on days zero (baseline), 15, 30, 45 and 60. The date were analyzed by ANOVA and Tuckey-Kramer test $(\alpha=0,05)$. The NAC did no exert no effect on the $\mathrm{U}-\mathrm{P} / \mathrm{C}$, however, the SBP of older dogs healthy there was a tendency to decrease form 15 days of treatment. It was concluded that NAC promoted in older dogs healthy a tendency to decrease SBP.
\end{abstract}

Keywords: Creatinine; Hypertension; Proteinuria.

\footnotetext{
${ }^{1}$ Docente do Centro Univesitário de Rio Preto (UNIRP) e FCAV - UNESP Jaboticabal-SP

${ }^{2}$ Docente do Departamento de Clínica e Cirurgia, FCAV/Unesp-Jaboticabal, Via de acesso "Prof. Dr. Paulo Donato Castellane" Km 5, Jaboticabal, SP 14884-900.
} 


\section{INTRODUÇÃO}

Quando se analisa o mecanismo fisiopatológico básico dos transtornos renais, observa-se a existência de fatores que predispõem ao desequilíbrio oxidativo. $\mathrm{O}$ estresse oxidativo é definido como o acúmulo de espécies reativas do oxigênio (ERO) que promovem danos às estruturas das biomoléculas de DNA, lipídios, carboidratos e proteínas, além de outros componentes celulares. Acredita-se que o estresse oxidativo seja um fator potencialmente importante na mortalidade dos pacientes com doença renal crônica (DRC) e na mediação de muitas complicações, pois está envolvido na patogênese da hipertensão arterial sistêmica (HAS), disfunção endotelial e inflamação (SCOTT, 2008).

Sabe-se que o paciente doente renal crônico apresenta-se mal nutrido e com carência de vitaminas e minerais, o que diminui os mecanismos de defesa antioxidantes, favorecendo o incremento do estresse oxidativo renal, com a formação de ERO (SCOTT, 2008). Contra a ação potencialmente lesiva destas substâncias reativas, torna-se fundamental o controle minucioso de sua produção e consumo no meio intracelular. Isto pode ser feito pelos antioxidantes, que, removendo as substâncias reativas, as mantêm em concentrações baixas (LAURINDO, 2003).

A N-acetilcisteína (NAC) é um antioxidante que tem sido empregado na proteção renal em modelos experimentais de insuficiência renal aguda isquêmica, NOS estudos da lesão renal aguda tóxica por contraste radiográfico em animais e humanos ou por outros fármacos nefrotóxicos em humanos e, também, na DRC (SHIMIZU et al., 2005).

Este antioxidante é um aminoácido, tiol, agente mucolítico e precursor da L-cisteina e glutationa nas células. Trata-se de uma fonte de grupos sulfidril removedores de ERO (LOCATELLI et al., 2003). No entanto, o efeito deste antioxidante na pressão arterial sistólica (PAS) e na excreção urinária de proteína (U-P/C) de cães idosos sadios e com DRC não foi avaliado, com este propósito foi conduzido o presente estudo.

\section{MATERIAL E MÉTODO}

Foram, então, selecionados 18 cães idosos ( 9 a 15 anos) sadios e animais com DRC naturalmente adquirida, sem infecção urinária ou outra doença concomitante e com quadro clínico estável, sendo incluídos cães com DRC conforme a Iris (2009). Os cães foram provenientes do canil e/ou do atendimento do Serviço de Nefrologia e Urologia Veterinária da Faculdade de Ciências Agrárias e Medicina Veterinária, Universidade Estadual Paulista Jaboticabal, SP. A categorização dos grupos foi estabelecida pela concentração sérica de creatinina (Scr) e ureia (Srueia) na avaliação basal. Os animais foram divididos em quatro grupos, 
normal controle $(\mathrm{N}-\mathrm{C} ; \mathrm{n}=4)$, normal tratado $(\mathrm{N}-\mathrm{T} ; \mathrm{n}=5)$, DRC controle (DRC-C; $\mathrm{n}=5$ ) e DRC tratado (DRC-T; $\mathrm{n}=4$ ). A média \pm erro padrão da Scr e Sureia dos grupos N-C e N-T foi 0,76 \pm $0,10 \mathrm{mg} / \mathrm{dL}, 18,01 \pm 2,12 \mathrm{mg} / \mathrm{dL}$ e $1,03 \pm 0,08 \mathrm{mg} / \mathrm{dL}, 21,98 \pm 5,53 \mathrm{mg} / \mathrm{dL}$, respectivamente. Nos grupos DRC-C e DRC-T as respectivas médias \pm erro padrão da Scr e Sureia foi de 2,46 \pm 0,40 $\mathrm{mg} / \mathrm{dL}, 88,90 \pm 13,39 \mathrm{mg} / \mathrm{dL}$ e 2,03 $\pm 0,49 \mathrm{mg} / \mathrm{dL}, 65,39 \pm 21,29 \mathrm{mg} / \mathrm{dL}$.

Os cães dos grupos N-T e DRC-T receberam como tratamento único a NAC na dose de 10mg/kg, V.O., b.i.d, durante 60 dias. Os cães dos grupos N-C e DRC-C não receberam qualquer tipo de tratamento.

$\mathrm{O}$ tratamento experimental foi feito com o antioxidante $\mathrm{N}$-acetilcisteína (Fluimucil® suspensão $40 \mathrm{mg} / \mathrm{mL}$ - Laboratório Zambon), administrado por via oral, na dose de $10 \mathrm{mg} / \mathrm{kg}$, b.i.d., durante 60 dias, nos grupos N-T e DRC-T. A dosagem foi estabelecida de acordo com o descrito por Papich (2009).

A condução do experimento seguiu um delineamento em blocos casualizados e, para cada animal, teve duração de 61 dias. Os parâmetros PAS e U-P/C foram avaliados no dia zero sendo feitas as primeiras avaliações (basal). Para os dois grupos que receberam o tratamento com NAC, a administração foi iniciada no dia um e seguiu, sem interrupção, até serem completados 60 dias. Todos os animais dos quatro grupos foram reavaliados aos 15, 30, 45 e 60 dias nas determinações da PAS e U-P/C.

Para a determinação da concentração sérica de creatinina e ureia sérica, as amostras de soro foram processadas método Jaffé modificado e método enzimático, respectivamente. Nas amostras de urina foram dosadas creatinina (método Jaffé modificado), proteína total urinária (método vermelho de pirogalol). Para as leituras utilizou-se espectrofotômetro (LABTEST - Labest Diagnóstica S.A., Lagoa Santa-MG) semi-automático.

A proteinúria foi avaliada por meio da determinação da razão proteína/ creatinina da urina (U-P/C), a partir dos valores de concentração de creatinina e de proteína obtidas na mesma amostra de urina.

Para a determinação da PAS, foi utilizado o aparelho doppler vascular (Doppler Vascular DV10 Pastilha Microem), dotado de módulo de coleta não-invasiva. Para cada avaliação foram realizadas sete mensurações consecutivas para obtenção de uma média mais acurada.

A utilização de animais, seguindo o protocolo experimental do presente estudo foi previamente aprovada, pela Comissão de Ética no Uso de Animais da Faculdade de Ciências Agrárias e Veterinárias Unesp - campus de Jaboticabal-SP conforme processo n. ${ }^{\circ}$ 006823-09.

O método estatístico utilizado para avaliar as respostas foi a analise de variância com medidas repetidas. Comparações de médias aos pares foram feitas usando o Teste de Tukey- 
Kramer. Para todas as análises adotou-se $\alpha=0,05$. As análises foram realizadas por meio do softwear SAS 9.1.3., SAS Institute Inc., USA.

\section{RESULTADOS}

Durante todo período de estudo os cães idosos sadios e com doença renal crônica se mantiveram clinicamente bem, não tendo sido observado quaisquer manifestações que pudessem sugerir problemas decorrentes dos procedimentos experimentais.

A PAS foi influenciada significativamente pelo fator doença e houve interação significativa entre tratamento e tempo. Considerando as médias gerais dos grupos, o valor observado no grupo DRC-C $(152 \pm 7,46 \mathrm{mmHg})$ foi significativamente maior do que o do N-T $(129 \pm 10,66 \mathrm{mmHg})$, não tendo sido constatadas outras diferenças significativas (Tabela 1). A PAS não variou significativamente ao longo do tempo dentro de cada grupo (Tabela 2). Contudo, deve ser salientada a tendência de diminuição da PAS a partir dos 15 dias, em todos os animais do grupo N-T.

A U-P/C foi influenciada significativamente pelos fatores tempo e doença, mas houve interação entre os três fatores e as médias gerais dos grupos não diferiram significativamente entre si (Tabela 1). Também não foram observadas variações significativas em função do tempo (basal, 15, 30, 45 e 60 dias), dentro de cada grupo (Tabela 2).

Tabela 1 - PAS e U-P/C - avaliação dos efeitos do tratamento com n-acetilcisteína ${ }^{\mathbf{1}}$. Média \pm desvio padrão e resultados da análise estatística (ANOVA, $\alpha=0,05$ ) de dados obtidos em cinco avaliações de cães normais controle $(\mathrm{N}-\mathrm{C} ; \mathrm{n}=4)$, cães normais tratados $(\mathrm{N}-\mathrm{T} ; \mathrm{n}=5)$, cães com doença renal crônica controle (DRC-C; $\mathrm{n}=5)$ e cães com doença renal crônica tratados (DRC-T, $\mathrm{n}=4)$.

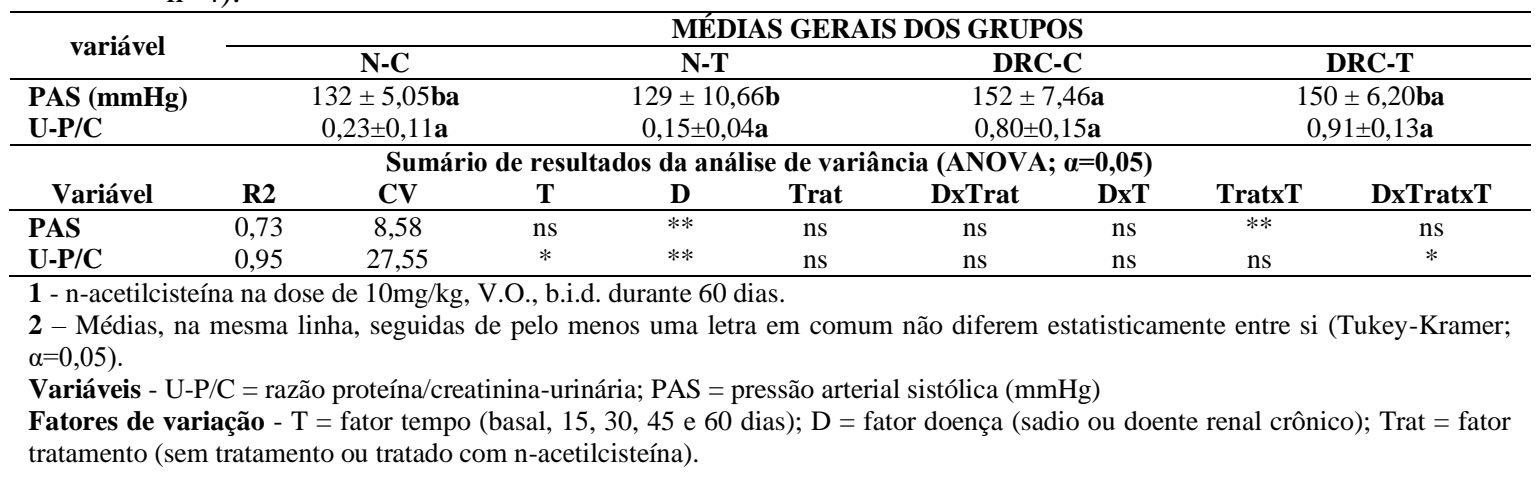


Tabela 2 - PAS e U-P/C - avaliação dos efeitos do tratamento com n-acetilcisteína ${ }^{\mathbf{1}}$. Média \pm desvio padrão de dados obtidos em cinco avaliações de cães normais controle $(\mathrm{N}-\mathrm{C} ; \mathrm{n}=4)$, cães normais tratados $(N-T ; n=5)$, cães com doença renal crônica controle (DRC-C; n=5) e cães com doença renal crônica tratados (DRC-T, $\mathrm{n}=4$ ).

\begin{tabular}{|c|c|c|c|c|c|}
\hline \multirow[b]{2}{*}{ variável } & \multirow{2}{*}{ tempo } & \multicolumn{4}{|c|}{ GRUPOS } \\
\hline & & $\mathrm{N}-\mathrm{C}$ & N-T & DRC-C & DRC-T \\
\hline \multirow{4}{*}{$\begin{array}{l}\text { Pressão arterial } \\
\text { sistólica } \\
(\mathrm{mmHg})\end{array}$} & basal & $125 \pm 4,08$ & $147 \pm 21,44$ & $142 \pm 22,49$ & $148 \pm 13,02$ \\
\hline & 30 dias & $131 \pm 14,18$ & $129 \pm 13,42$ & $150 \pm 11,67$ & $155 \pm 8,43$ \\
\hline & 45 dias & $134 \pm 9,52$ & $122 \pm 12,76$ & $157 \pm 22,29$ & $142 \pm 3,30$ \\
\hline & 60 dias & $139 \pm 10,69$ & $123 \pm 13,96$ & $161 \pm 21,30$ & $148 \pm 15,78$ \\
\hline \multirow{4}{*}{$\mathrm{U}-\mathrm{P} / \mathrm{C}$} & 15 dias & $0,42 \pm 0,23$ & $0,13 \pm 0,06$ & $0,73 \pm 0,62$ & $1,01 \pm 0,56$ \\
\hline & 30 dias & $0,19 \pm 0,10$ & $0,16 \pm 0,05$ & $0,77 \pm 0,66$ & $0,83 \pm 0,41$ \\
\hline & 45 dias & $0,14 \pm 0,04$ & $0,18 \pm 0,06$ & $0,75 \pm 0,72$ & $0,89 \pm 0,51$ \\
\hline & 60 dias & $0,20 \pm 0,11$ & $0,10 \pm 0,02$ & $0,86 \pm 0,68$ & $0,72 \pm 0,68$ \\
\hline
\end{tabular}

1 - n-acetilcisteína na dose de $10 \mathrm{mg} / \mathrm{kg}$, V.O., b.i.d. durante 60 dias.

\section{DISCUSSÃO}

Segundo Grauer (2007) e IRIS (2009), valores da U-P/C normais estão entre 0,2 a 0,3 ou menos, para a espécie canina. Quando presente em cães, a proteinúria persistente com valores da U-P/C entre 0,4 e 0,5 ou superiores, descartada a possibilidade de proteinúria pré-renal ou pósrenal, pode ser considerada um sinal de DRC glomerular ou túbulo-intersticial. Nos animais do presente estudo os valores de U-P/C dos cães com DRC-C e DRC-T foram maiores do que os dos grupos N-C e N-T, e embora não tenha havido diferença significativa entre as médias houve influência significativa do fator doença. Outrossim, os valores observados são clinicamente significativos para diagnóstico de proteinúria. O tratamento com NAC parece não ter influenciado a proteinúria no presente estudo, como também observado por Shimizu et al. (2005), que obteve resultados semelhantes, em ratos com DRC induzida, tratados com NAC por 120 dias via oral.

De acordo com Bartges et al. (1996), em cães com doença glomerular, a HAS é detectada em $85 \%$ dos casos. Segundo estudo desenvolvido por Jacob et al. (2003) a detecção de PAS aumentada por ocasião do diagnóstico inicial da DRC em cães, esta associada com aumento dos riscos de ocorrência de crise urêmica, morte e declínio rápido da função renal. No presente estudo a PAS foi influenciada significativamente pelo fator doença, mas também houve interação entre tratamento e tempo, resultando em inconsistência dos resultados de comparação de médias. Do ponto de vista clínico, parece adequado afirmar que a PAS dos pacientes doentes renais crônicos eram maiores do que a dos cães normais. Outro ponto a ser destacado por razões clínicas é referente à ação da NAC nos cães normais. Os dados representados (Tabela 2) demonstram que todos os cães responderam com diminuição da PAS detectada aos 15, 30, 45 e 60 dias de 
tratamento com NAC. O mesmo tipo de efeito, entretanto, não foi observado nos cães do grupo DRC-T. Song et al. (2005) estudaram o efeito da administração da NAC em ratos normais tratados com frutose, substância esta que induz aumento da PAS e resistência à insulina. Observaram, então, que o grupo que recebeu frutose e NAC apresentou a PAS e a pressão arterial diastólica menor do que as do grupo que recebeu somente frutose. Em um estudo realizado por Rauchová et al. (2005) com N-nitro-L-arginine metil ester, fármaco este, que eleva a pressão arterial sistêmica, foi demonstrado que ratos normais quando foram submetidos ao tratamento com N-nitro-L-arginine metil ester, apresentaram a pressão arterial sistêmica elevada, quando comparados ao outro grupo de ratos tratados com N-nitro-L-arginine metil ester e NAC, que apresentaram pressões menores, evidenciando, assim, o efeito hipotensor do antioxidante NAC. Ambos os autores atribuíram o efeito à provável redução das ERO e ao aumento da vasodilatação por acréscimo da viabilidade de óxido nítrico no parênquima renal. Boesgaard et al. (1993) demonstraram a ação da NAC na enzima conversora de angiotensina, em ratos normais que foram submetidos ao tratamento com o antioxidante NAC intravenoso. Este grupo, diferentemente do grupo placebo, apresentou redução da enzima conversora de angiotensina em $31 \%$ no plasma e $43 \%$ no parênquima renal. Shimizu et al. (2005) submeteu ratos com DRC induzida, ao tratamento com NAC, via oral por 120 dias, e não observou diferença na pressão arterial dos animais tratados em relação aos animais controle. Semelhantemente, nossos resultados mostraram que a administração de NAC não causou redução da PAS em cães com DRC.

\section{CONCLUSÃO}

Conclui-se que a NAC não exerceu efeito nenhum sobre a U-P/C, entretanto, na PAS de cães idosos sadios houve uma tendência a redução a partir de 15 dias de tratamento.

\section{AGRADECIMENTOS}

Ao Conselho Nacional de Desenvolvimento Científico e Tecnológico (CNPq) pela concessão do auxílio à pesquisa CNPq n. ${ }^{\circ} 474017 / 2008-7$; ao Serviço de Nefrologia e Urologia Veterinária do Departamento de Clínica e Cirurgia Veterinária - Unesp- campus de JaboticabalSP pela concessão dos animais; a Faculdade de Ciências Agrárias e Veterinárias - Unesp campus de Jaboticabal-SP pela concessão dos laboratórios e de toda infraestrutura. 


\section{REFERÊNCIAS}

BARTGES, J. W. Hypertension and Kidney disease. Veterinary Clinics of North America Small Animal Medicine Practice, New York, v. 26, n. 6, p. 1331-1345, 1996.

BOESGAARD, S.; ALERSHVILE, H.; POULSEN, E.; CHRISTENSEN, H.; PETERSEN, D.; GIESE, J. N-acetyl cysteine inhibits angiotensin converting enzyme in vivo. The Journal of Pharmacology and Experimental Therapeutics, v. 265, n. 3, p. 1239-1244, 1993.

GRAUER, G.F. Measurement, Interpretation, and Implications of Proteinuria and Albuminuria Veterinary Clinics of North American Small Animal Practice. New York, v. 37, n. 2, p. 283 295, 2007.

IRIS. International Renal Interest Society. Staging Chronic Kidney Disease (CKD) 2009. Disponível em: 〈http://www.iris-kidney.com/pdf/IRIS\%20A4\%20Poster.pdf >. Acessado em: 14 abr. 2013.

JACOB, F. POLZIN, D. J. OSBORNE, C. A. NEATON, J. D.; LEKCHAROENSUK, C. ALLEN, T. A.; KIRK, C. A.; SWANSON, L. L. Association between initial systolic blood pressure and risk of developing a uremic crisis or of dying in dogs with chronic renal failure. Journal of the American Veterinary Medical Association, Schaumburg, v. 222, n. 3, p. 322329, 2003.

LAURINDO, F. R. M. Desequilíbrio Redox. Resposta Cardiovascular à lesão da aterosclerose. Endotélio e Doenças Cardiovasculares. Da Luz L. P., Laurindo, Chagas CPA, São Paulo: Atheneu. 2003, cap. 8, p. 97-113.

LOCATELli, F.; CANAUD, B.; ECKARDT, K. U.; STENVINKEL, P.; WANNER, C., ZOCCATLI, C. Oxidative stress in end-stage renal disease: an emerging threat to pacient outcome. Nephrology Dialysis Transplantation, Oxford, v. 18, n. 7,p. 1272-1280, 2003.

PAPICH, M. G. Saunders Handbook of Veterinary Drugs. 2 th. St Louis: Elsevier, 2009, $774 \mathrm{p}$.

RAUCHOVÁ, H.; PECHANOVÁ, O.; KUNES, J.; VOKURKOVÁ, M.; DOBESOVÁ, Z.; ZICHA, J. Chronic N-acetylcysteine administration prevents development of hypertension in Nnitro-L-arginine methyl Ester-treated rats: the role of reactive oxygen species. Kidney International, New York, v. 63, p. 634-341, 2005.

SCOTT, A. N. D. Oxidative stress and chronic kidney disease. Veterinary Clinics of North American Small Animal Practice, New York, v. 38, n. 1, p. 157-166, 2008.

SHIMIZU, M. H.; COIMBRA, T. M.; ARAÚJO, M. et al. N-acetylcysteine attenues the progression of chronic renal failure. Kidney International, New York, v. 68, p. 2208-217, 2005.

SONG. D.; HUTCHINGS, S.; PANG, C. Chronic N-acetylcysteine prevents fructose-induced insulin resistance and hypertension in rats. The European Journal of Pharmacology, Oxford, $\mathrm{v}$ 508, n. 3, p. 205-210, 2005. 